\title{
Entrenamiento mediante sprints repetidos en futbolistas: Efectos sobre la capacidad de repetir sprint, salto y tiempo de reacción
}

\author{
Repeated sprints training in soccer players: Effects on repeated sprint ability, \\ jump and reaction time \\ Iván Asín Izquierdo ${ }^{1}$, Luis Gutiérrez García ${ }^{1}$, Javier Raya-González ${ }^{2}$, Daniel Castillo² \\ Javier Sánchez-Sánchez ${ }^{3}$, Alejandro Rodríguez Fernández ${ }^{4}$ \\ 1 Departmento de Ciencias Biomédicas. Universidad de Alcalá. Madrid. España. \\ 2 Facultad de Ciencias de la Salud. Universidad Internacional Isabel I. Burgos. España. \\ 3 Facultad de Educación. Universidad Pontificia de Salamanca. Salamanca. España. \\ 4 Departamento de Educación Física y Deportiva. Universidad de León. León. España.
}

CORRESPONDENCIA:

Luis Gutiérrez García

luis.gutierrezgarcia@gmail.com

Recepción: septiembre 2019 • Aceptación: diciembre 2020

\section{CÓMO CITAR EL ARTÍCULO:}

Asín Izquierdo, I., Gutiérrez-García, I., Raya-González, J., Castillo, D., Sánchez-Sánchez, J., \& Rodríguez Fernández, A. (2021). Entrenamiento mediante sprints repetidos en futbolistas: Efectos sobre la capacidad de repetir sprint, salto y tiempo de reacción. Cultura, Ciencia y Deporte, 16(49), 337-345. http://dx.doi.org/10.12800/ ccd.v16i49.1264

\section{Resumen}

El entrenamiento de sprint repetidos parece ser una estrategia eficiente para el desarrollo simultáneo de diferentes componentes de la condición física en deportes intermitentes. El objetivo del presente estudio fue analizar los efectos de suplementar el entrenamiento habitual, mediante un entrenamiento basado en sprint repetidos, sobre la capacidad de repetir sprints (RSA), el tiempo de reacción en situación de fatiga y la capacidad de salto. Para ello veintisiete jóvenes futbolistas (edad $17.7 \pm 0.7$ años) fueron divididos en dos grupos y asignados a un grupo de entrenamiento (GER, $n=14$ ) y a un grupo control $(G C, n=13)$. Ambos grupos realizaron cuatro semanas de idéntico entrenamiento, para posteriormente realizar cuatro semanas de la intervención específica GER (entrenamiento habitual más 3 sesiones semanales realizando 2 series de 8 repeticiones sobre 10-30 metros a la máxima intensidad) y continuar con el entrenamiento habitual GC durante las 4 últimas semanas de la temporada. No se mostraron efectos significativos en la mejora del tiempo medio ( $T E=-0.17$; Posible Trivial) y el decrecimiento ( $T E=$ -0.11; poco claro) del test RSA, ni en la diferencia entre el tiempo de reacción del sprint 1 y 8 de un test RSA antes y después de la intervención ( $T E=0.27$; poco claro) mediante sprint repetidos. La suplementación del entrenamiento mediante sprint repetidos al final del macrociclo competitivo no mostró efectos significativos en la mejora del RSA ni en el tiempo de reacción en jóvenes futbolistas.

Palabras clave: Resistencia a la velocidad, temporada competitiva, deportes de equipo, rendimiento, capacidad de repetir sprint (RSA).

\section{Abstract}

Repeated sprint training appears to be an efficient strategy for the simultaneous development of different fitness components in intermittent sport. The aim of this study was to analyze the effects of implement training with repeated sprint training on repeated sprint ability (RSA), reaction time in fatigue condition and jump height. Twenty-seven young soccer players (age $17.7 \pm 0.7$ years) were assigned to intervention group (GER, $n=14$ ) and control group $(G C, n=13)$. Both groups perform over four weeks the identical training program with the objective that the previous training did not condition the results and later, GER implement the habitual training with specific intervention (habitual training plus 3 weekly sessions, 2 sets of 8 repetitions on 10-30 meters at maximum intensity) and GC continue with habitual training over the last four weeks of the competitive period. Repeated sprint training not shown significant improvement in mean time ( $T E=-0.17$, Possible Trivial) and decrement (TE $=-0.11$, unclear) obtained in an RSA test, nor in the difference between the reaction time of sprint 1 and 8 of an RSA test before and after the intervention ( $T E=0.27$; Clear). The implementation of the habitual training by RST at the end of the competitive season has not shown significant positive effects in the RSA nor in the reaction time in young players.

Key words: specific endurance, season, team sport, performance, repeated sprint ability. 


\section{Introducción}

El fútbol es un deporte con requerimientos condicionales, psicológicos, técnicos y tácticos manifestados de forma conjunta. Si atendemos a las demandas condicionales, jugadores jóvenes de fútbol (U18-U20) recorren una distancia total de 8000-9000 m (Buchheit, Méndez-Villanueva, Simpson, \& Bourdon, 2010; Pereira Da Silva, Kirkendall, \& Leite De Barros Neto, 2007) y una distancia relativa de entre 97.26-127.31 $\mathrm{m} / \mathrm{min}$ (Aughey et al., 2013; Vigh-Larsen, Dalgas, \& Andersen, 2018), alcanzando velocidades máximas de $28.3 \mathrm{~km} / \mathrm{h}$ (Buchheit, Méndez-Villanueva, Simpson, et al., 2010). Los jugadores deben realizar esfuerzos de naturaleza intermitente, caracterizados por periodos largos de actividad a baja intensidad, entre los que se intercalan de manera aislada y repetida acciones de alta intensidad (e.g., sprints) (Buchheit, Méndez-Villanueva, Simpson, et al., 2010). Estos esfuerzos suelen preceder a momentos cruciales del partido (Faude, Koch, \& Meyer, 2012) denominándose como worst case scenarios (Gabbett et al., 2016). Estos eventos son las situaciones más exigentes de la competición, con una gran demanda metabólica y neuromuscular (Méndez-Villanueva, Hamer, \& Bishop, 2007, 2008). Las secuencias de esfuerzos de alta intensidad y corta duración con breves periodos de recuperación se han denominado Repeated Sprint Ability (RSA) (Bishop, Girard, \& Méndez-Villanueva, 2011; Girard, MéndezVillanueva, \& Bishop, 2011). Aunque estudios previos cuestionan la importancia del RSA en el rendimiento en jóvenes jugadores de fútbol (Buchheit, Méndez-Villanueva, Simpson, \& Bourdon, 2010), otros estudios la consideran una capacidad determinante del rendimiento (Iaia et al., 2015), ya que diferencia el nivel competitivo de los jugadores (Impellizzeri et al., 2008; Rampinini et al., 2009). Se ha mostrado que existe una correlación $(r=-0.60, \mathrm{R} 2=0.36$; $\mathrm{p}<0.01)$ entre la distancia recorrida a alta intensidad durante el partido y el tiempo medio obtenido en un test RSA (Rampinini, Bishop, et al., 2007). Además, el fútbol está evolucionando con un aumento en las demandas físicas, mostrando un mayor número de sprints (85\%), distancia a sprint $(35 \%)$ y distancia total $(2 \%)$ entre la temporada 2006-2007 y 2012-2013, exigiendo a los jugadores una mayor repetición de esfuerzos a alta intensidad (Barnes, Archer, Hogg, Bush, \& Bradley, 2014). Estas demandas no son uniformes durante toda la temporada competitiva en futbolistas profesionales en Europa, debido a que en la parte final de la temporada los jugadores recorren una mayor distancia a alta ( $>14$ $\mathrm{km} / \mathrm{h}$ ) y muy alta intensidad ( $>19.8 \mathrm{~km} / \mathrm{h}$ ) que durante la parte media de la temporada (Rampinini, Coutts,
Castagna, Sassi, \& Impellizzeri, 2007). Por esta razón, los preparadores físicos deben manejar aquellos métodos que mejor puedan incidir sobre el rendimiento en aquellas situaciones más exigentes en función del momento de la temporada (Taylor, Macpherson, Spears, \& Weston, 2015).

En la literatura existen diferentes estudios que se han ocupado de analizar el efecto de distintos tipos de entrenamiento sobre RSA, como el entrenamiento mediante juegos reducidos (Owen, Wong del, Paul, \& Dellal, 2012; Rodríguez-Fernández, Sánchez Sánchez, Rodríguez-Marroyo, Casamichana, \& Villa, 2017), sprint repetidos (Buchheit, Méndez-Villanueva, Delhomel, Brughelli, \& Ahmaidi, 2010; Campos-Vazquez et al., 2015; Eniseler, Şahan, Özcan, \& Dinler, 2017), basado en la fuerza (Buchheit, Méndez-Villanueva, Delhomel, et al., 2010) o el entrenamiento de alta intensidad (Ferrari Bravo et al., 2008; Rodríguez Fernández, Sánchez Sánchez, \& Villa Vicente, 2014). Aunque algunos trabajos de revisión han aportado indicaciones generales para desarrollar el entrenamiento de esta capacidad (Bishop et al., 2011), en la actualidad no se conoce cuál es la estrategia más efectiva para la mejora del RSA (Buchheit, 2012). Entre las posibilidades existentes se encuentra el entrenamiento mediante sprint repetidos (RST), basado en la aplicación de series de sprints cortos (3-7 segundos), separadas por recuperaciones breves (<60 segundos) (M Buchheit \& Laursen, 2013).

Estudios previos han detectado mejoras en el RSA tras la aplicación de entrenamientos basados en RST (Buchheit, Méndez-Villanueva, Delhomel, et al., 2010; Rodríguez Fernández et al., 2014). Ferrai Bravo et al. (2008) mostraron que una intervención de 3 series de 6 sprints máximos de 40 metros es útil para mejorar el tiempo medio en un test RSA $(7.53 \pm 0.21$ a $7.37 \pm$ $0.17 \mathrm{~s})$ tras 7 semanas de entrenamiento. Por su parte, Eniseler et al. (2017) observaron que 3 series de 6 repeticiones de 40 metros con 20 segundos de recuperación, realizadas 2 veces por semana durante 6 semanas, no originó una mejora en el tiempo medio, pero si en el decrecimiento $(5.80 \pm 1.05 \%$ a $3.75 \pm 1.16 \%)$. Las mejoras a partir de la aplicación de RST pueden ser debidas a la estimulación de los factores limitantes del rendimiento de esta capacidad (e.g. resíntesis de fosfocreatina, metabolismo aeróbico y anaeróbico, acumulación de $\mathrm{H}^{+}$y activación muscular) (Bishop et al., 2011) o a la especificidad, que permite abordar directamente los factores limitantes del rendimiento en RSA (Taylor et al., 2015). No obstante, el alcance de este estímulo podría ser dependiente del momento de la temporada, debido a la relación entre la condición física del futbolista y la mejora en el RSA (Rodríguez- 
Fernández et al., 2017). Aunque el rendimiento en RSA disminuye levemente en los futbolistas a medida que avanza la competición, (i.e., inicio vs final de temporada) (Impellizzeri et al., 2008), el RST se ha mostrado útil para la mejora de esta capacidad tanto durante la pretemporada (Rodríguez Fernández et al., 2014) como durante el periodo competitivo (Buchheit, Méndez-Villanueva, Delhomel, et al., 2010; Ferrari Bravo et al., 2008). Este tipo de entrenamiento no ha mostrado efectos sobre la capacidad de salto tras la aplicación de un programa de entrenamiento basado en sprint repetidos de 10 (Buchheit, MéndezVillanueva, Delhomel, et al., 2010) o 12 semanas de duración (Ferrari Bravo et al., 2008).

En los deportes colectivos, los jugadores continuamente experimentan cambios contextuales, requiriendo de una percepción de la situación, procesamiento del estímulo y toma de decisión lo más rápidos posible (Baker, Cote, \& Abernethy, 2003), teniendo que reaccionar en muchas ocasiones en situación de fatiga. El término tiempo de reacción es ampliamente utilizado para referirse al tiempo que un sujeto tarda en responder a un estímulo ambiental (Wilkerson, Simpson, \& Clark, 2017). Este tiempo depende de la complejidad interpretativa que requieren los estímulos y los criterios usados para definir el inicio o completar la respuesta (Eckner, Kutcher, \& Richardson, 2010; Miller \& Low, 2001; Schwab \& Memmert, 2012). El tiempo de reacción simple hace referencia a aquel tiempo en el que el sujeto tiene que iniciar una respuesta motora o ejecutar una tarea simple, en respuesta a un estímulo visual o auditivo (Miller \& Low, 2001). Diferentes estudios han analizado el tiempo de reacción ante acciones generales (e.g. contactar un botón que se ilumina) (Miller \& Low, 2001) o mediante el uso de aparatos clínicos (Eckner et al., 2010), pero ningún estudio ha analizado la evolución del tiempo de reacción entre los diferentes sprint que componen un test RSA y la posible incidencia de la fatiga en el mismo mediante el patrón motor más específico del fútbol, como es la carrera.

No existen estudios que hayan analizado los efectos de un programa de entrenamiento mediante RST en el rendimiento en RSA y tiempo de reacción, aplicado en la última fase de la temporada competitiva. Por ello, el objetivo principal de este estudio fue analizar los efectos sobre el RSA, el tiempo de reacción y capacidad de salto de un entrenamiento RST, aplicado durante las cuatro últimas semanas del macrociclo competitivo a jugadores jóvenes de fútbol. La hipótesis principal de este estudio fue que la suplementación del entrenamiento habitual con entrenamiento basado en sprint repetidos mejoraría el rendimiento del futbolista en la capacidad de repetir sprints, salto y el tiempo de reacción.

\section{Método}

\section{Participantes}

Veintisiete futbolistas jóvenes (edad $17.7 \pm 0.7$ años, estatura $176.8 \pm 6.3 \mathrm{~cm}$, masa corporal $69.9 \pm 8.7 \mathrm{~kg}$ ) pertenecientes a dos equipos de categoría juvenil participaron en el estudio. Todos los participantes tenían una experiencia mínima de 6 años en la práctica del fútbol. Los jugadores entrenaban 3 veces a la semana (lunes, miércoles y viernes) y jugaban un partido de competición oficial los sábados. Antes del comienzo del estudio los participantes fueron informados de los posibles riesgos, objetivos y procedimientos del estudio, así como de los derechos y obligaciones vinculados a la participación. Los jugadores y los padres o tutores de los jugadores menores de edad firmaron un consentimiento informado, aceptando los procedimientos ligados al estudio. El diseño experimental se llevó a cabo de acuerdo al código de ética de la Declaración de Helsinki.

\section{Diseño experimental}

Se evaluaron los efectos de un entrenamiento RST de 12 sesiones sobre el rendimiento en un test RSA, el tiempo de reacción y la capacidad de salto en jugadores jóvenes de fútbol durante las últimas 4 semanas de la temporada competitiva. Los futbolistas de dos equipos de igual nivel competitivo fueron asignados al azar al grupo de entrenamiento mediante sprint repetidos (GER, $\mathrm{n}=14,3$ defensas centrales, 2 defensas laterales, 5 mediocentros, y 4 delanteros) o al grupo de entrenamiento habitual que fue considerado grupo control (GC, $\mathrm{n}=13$, 4 defensas centrales, 1 defensa lateral, 3 mediocentros, y, 5 delanteros). Ambos grupos realizaron el mismo entrenamiento de fútbol durante las 4 semanas previas a la intervención, realizando 3 sesiones por microciclo junto con un partido habitual. Posteriormente, coincidiendo con las 4 últimas semanas de competición, se desarrolló el período de intervención, donde GER suplementó el entrenamiento específico de fútbol con el RST. Las variables dependientes de investigación (Tiempo medio, tiempo total y decrecimiento en el test RSA, altura de salto y tiempo de reacción) fueron evaluadas antes (pre-test) y después (post-test) del período de intervención.

\section{Procedimiento}

\section{Intervención}

El trabajo se desarrolló durante 8 semanas, correspondientes a los 2 últimos meses del periodo compe- 
titivo. Durante las primeras 4 semanas se realizaron 2 sesiones de familiarización con el entrenamiento RST y con los test de evaluación del rendimiento. En este período GER y GC realizaron 3 sesiones de entrenamiento de 90 minutos por semana, con la misma estructura de microciclo (Owen, Lago-Peñas, Gómez, Mendes, \& Dellal, 2017), carga y actividades: lunes, entrenamiento aeróbico de moderada intensidad y trabajo de prevención de lesiones; miércoles, entrenamiento de potencia aeróbica, juegos reducidos (i.e. 4 vs. 4 en 25 x 20 m y 5 vs. 5 en 40 x 35 m) y simulación de competición (11 vs 11 en campo completo); viernes, entrenamiento de velocidad y acciones combinativas de finalización. Durante el segundo mesociclo se mantuvo el mismo entrenamiento de fútbol, pero GER realizó de forma intercalada entre las actividades que componían la sesión tareas específicas de RST. La semana anterior y posterior a este mesociclo se desarrolló una sesión de evaluación para analizar el efecto del entrenamiento diseñado sobre la condición física de los jugadores. Antes del test RSA los jugadores realizaron un calentamiento estandarizado dirigido por el mismo técnico especialista, que incluía ejercicios de movilidad articular, estiramientos dinámicos y 3 repeticiones de sprint progresivo de $30 \mathrm{~m}$. Los test se realizaron en el campo de hierba artificial donde cada equipo realizó su entrenamiento diario, con la indumentaria y calzado habitual de práctica. Durante el desarrollo de la fase experimental se pidió a los jugadores que mantuviesen sus hábitos de vida normal, que incluían 8 horas de sueño nocturno, hidratación óptima y completa ingesta de hidratos de carbono. El entrenamiento RST realizado durante 12 sesiones por GER, como complemento al entrenamiento habitual de fútbol, consistió en la realización de 2 series de 8 repeticiones sobre 10-30 metros a la máxima intensidad posible (Tabla 1 ). Un técnico especialista con 5 años de experiencia en el entrenamiento del fútbol se encargó de la supervisión de la ejecución y de animar a los jugadores para que realizarán las series a la máxima intensidad.

\section{Valoración del rendimiento}

Capacidad para repetir sprints (RSA). Tras la realización de un calentamiento estandarizado se evaluó el RSA realizando 8 sprints de 30 metros con 25 segundos de recuperación (Rodríguez-Fernández et al., 2017). El criterio de maximalidad se basó en la selección del mejor sprint de 3 posibles intentos realizados 5 minutos antes del comienzo del test y con 180 segundos de recuperación entre cada uno de ellos (Impellizzeri et al., 2008). Cuando el tiempo del primer sprint del test RSA era un $2.5 \%$ peor que el tiempo del sprint
Tabla 1. Características del programa de entrenamiento mediante sprint repetidos realizado por el grupo de intervención (GER) suplementado a su entrenamiento habitual.

\begin{tabular}{|c|c|c|c|}
\hline & Lunes & Miércoles & Viernes \\
\hline Series & 2 & 2 & 2 \\
\hline Repeticiones & 8 & 8 & 8 \\
\hline $\begin{array}{l}\text { Recuperación entre } \\
\text { repeticiones } 1^{\text {a }} \text { serie (s) }\end{array}$ & 15 & 20 & 10 \\
\hline $\begin{array}{l}\text { Recuperación entre } \\
\text { repeticiones } 2^{\mathrm{a}} \text { serie (s) }\end{array}$ & 8 & 10 & 8 \\
\hline Distancia $1^{\text {a }}$ serie $(\mathrm{m})$ & 20 & 30 & 15 \\
\hline Distancia $2^{\mathrm{a}}$ serie $(\mathrm{m})$ & 10 & 15 & 10 \\
\hline Total (m) & 400 & 360 & 200 \\
\hline
\end{tabular}

utilizado como criterio de maximalidad el test se daba por finalizado y el jugador debía realizar un nuevo intento 5 minutos después (Impellizzeri et al., 2008). Durante la ejecución del test los jugadores salían desde una señal colocada 0.5 metros antes de la primera fotocélula (Sistema telemétrico de cronometraje Bosco System, Ergotests Technology, Cronómetro digi sport instruments DT 8000, Noruega) para evitar falsos cortes (Chaouachi et al., 2010), y corrían lo más rápido posible hasta la siguiente fotocélula. Una vez cruzada esta referencia regresaban al punto de inicio realizando carrera de baja intensidad. Un marcador luminoso (Ergotests Technology, Noruega) avisaba a los jugadores a través de una cuenta atrás del tiempo que faltaba para el comienzo del siguiente sprint, apareciendo una señal luminosa y auditiva para indicar la salida. Los futbolistas fueron fuertemente motivados para ofrecer un esfuerzo máximo durante toda la evaluación. Las variables utilizadas para determinar el rendimiento en el test RSA fueron el mejor tiempo $\left(\mathrm{RSA}_{\text {mejor }}\right)$ y el tiempo medio de los 8 sprints $\left(\mathrm{RSA}_{\text {media }}\right.$ ) (Fransson et al., 2018). Para cuantificar la fatiga se utilizó el porcentaje de decrecimiento (Sdec) calculado como Sdec $=\left(\mathrm{RSA}_{\text {total }} /\left(\mathrm{RSA}_{\text {mejor }} \times 8\right)^{-1} \times 100\right)-100$ (Girard et al., 2011). Además, el Sdec modificado (MSdec) fue calculado, teniendo en cuenta el mejor sprint del test RSA obtenido en ambas valoraciones para determinar el decrecimiento $\left(\mathrm{Sdec}=\left(\mathrm{RSA}_{\text {total }} / \mathrm{RSA}_{\text {mejor en valoración pre y }}\right.\right.$ post intervención $\left.\left.\times 8)^{-1} \times 100\right)-100\right)$.

Capacidad de salto del tren inferior. La valoración de la capacidad de salto se realizó mediante la medición del salto vertical Counter Movement Jump (CMJ) con la aplicación móvil MyJump ${ }^{\circledR}$ (Balsalobre-Fernández, Glaister, \& Lockey, 2015). Previamente a la realización del test RSA se realizó la prueba CMJ. Cada salto fue validado mediante la inspección visual para asegurar que el aterrizaje se realizaba sin flexión de las piernas. Los jugadores fueron instruidos para mantener las 
Tabla 2. Cambios en la capacidad de salto, sprint repetidos y tiempo de reacción en el grupo intervención (GER, $n=14)$ y el grupo control $(\mathrm{GC}, n=13)$ tras 8 semanas de intervención.

\begin{tabular}{|c|c|c|c|c|c|c|c|}
\hline Variables & Grupo & Pre-test & Post-test & $\Delta \% ; \pm 90 \%$ LC & $\mathrm{TE} ; \pm 90 \% \mathrm{LC}$ & Valoración cualitativa & Probabilidad \\
\hline \multirow[t]{2}{*}{$\mathrm{CMJ}(\mathrm{cm})$} & GER & $30.85 \pm 4.33$ & $33.75 \pm 4.15$ & $2.9 ; \pm .6$ & $0.63 ; \pm 0.14$ & $\begin{array}{l}\text { Extremadamente Probable } \\
\text { Moderado }\end{array}$ & $100 / 0 / 0$ \\
\hline & $\mathrm{GC}$ & $32.25 \pm 4.32$ & $32.82 \pm 4.90$ & $.6 ; \pm .8$ & $.12 ; \pm .17$ & Improbable Pequeño & $22 / 78 / 0$ \\
\hline \multirow[t]{2}{*}{$\mathrm{RSA}_{\text {mejor }}(\mathrm{s})$} & GER & $4.62 \pm .26$ & $4.58 \pm .20$ & $.0 ; \pm .1$ & $-.15 ; \pm .23$ & Posible Trivial & $1 / 65 / 34$ \\
\hline & $\mathrm{GC}$ & $4.55 \pm .25$ & $4.47 \pm .27$ & $-.1 ; \pm .0$ & $-.30 ; \pm .17$ & Probable Pequeño & $0 / 18 / 82$ \\
\hline \multirow[t]{2}{*}{$\mathrm{RSA}_{\text {media }}(\mathrm{s})$} & GER & $4.84 \pm .30$ & $4.79 \pm .25$ & $-.1 ; \pm .1$ & $-.17 ; \pm .16$ & Posible Trivial & $0 / 62 / 38$ \\
\hline & GC & $4.82 \pm .28$ & $4.75 \pm .31$ & $-.1 ; \pm .1$ & $-.25 ; \pm .22$ & Posible Pequeño & $0 / 34 / 66$ \\
\hline \multirow[t]{2}{*}{ Sdec (\%) } & GER & $4.79 \pm 2.21$ & $4.53 \pm 1.53$ & $-.3 ; \pm 1.2$ & $-.11 ; \pm .49$ & Poco claro & $14 / 48 / 37$ \\
\hline & GC & $5.91 \pm 1.85$ & $6.04 \pm 2.15$ & $.1 ; \pm 1.0$ & $.06 ; \pm .52$ & Poco claro & $33 / 48 / 19$ \\
\hline \multirow[t]{2}{*}{ MSdec (\%) } & GER & $6.48 \pm 2.20$ & $5.37 \pm 1.97$ & $-1.1 ; \pm 1.1$ & $-.48 \pm .48$ & Probable Pequeño & $1 / 15 / 84$ \\
\hline & $\mathrm{GC}$ & $8.07 \pm 2.90$ & $6.26 \pm 2.14$ & $-1.8 ; \pm 1.6$ & $-.59 ; \pm .51$ & Probable Pequeño & $1 / 9 / 90$ \\
\hline \multirow[t]{2}{*}{ TReacc (\%) } & GER & $-6.06 \pm 17.84$ & $-1.0 \pm 12.86$ & $5.1 ; \pm 12.5$ & $0.27 ; \pm .66$ & Poco claro & $57 / 31 / 12$ \\
\hline & $\mathrm{GC}$ & $5.04 \pm 39.99$ & $8.02 \pm 25.09$ & $3.0 ; \pm 9.7$ & $.07 ; \pm .23$ & Improbable Trivial & $16 / 81 / 3$ \\
\hline
\end{tabular}

Nota: $\Delta \%$ = porcentaje de cambio entre pre y post intervención; LC = límites de confianza; TE = tamaño del efecto; CMJ = salto con contramovimiento; $\mathrm{RSA}_{\text {mejor }}=$ mejor tiempo de los 8 sprints del test RSA; RSA ${ }_{\text {media }}$ tiempo medio de los 8 sprints del test RSA; Sdec =Índice de decrecimiento; MSdec = Índice de decrecimiento modificado; Treacc = porcentaje de cambio en tiempo de reacción entre la repetición 1 y 8 del test RSA.

manos en su cintura durante la realización del salto. El CMJ fue realizado tres veces con una recuperación pasiva de 45 segundos registrando el mejor salto.

Tiempo de reacción. Durante la realización del test RSA, por medio del registro del tiempo de reacción simple de los jugadores, se valoró el tiempo de reacción. Para ello, antes de cada uno de los 8 sprints que componían el test RSA, se registró el tiempo que transcurría desde la salida a 0.5 metros hasta la primera fotocélula (Sistema telemétrico de cronometraje Bosco System, Ergotests Technology, Cronómetro digi sport instruments DT 8000, Noruega). Para analizar el efecto del entrenamiento se utilizó el tiempo de reacción obtenido en la primera y última repetición del test calculado como porcentaje de cambio (TReacc $=$ (Tiempo de reacción sprint 8 - Tiempo de reacción sprint 1) / Tiempo de reacción sprint 1) y el tiempo absoluto de las repeticiones 1 y 8 .

\section{Análisis estadístico de los datos}

Los resultados se presentan como media \pm desviación estándar $(S D)$. Todos los datos fueron transformados logarítmicamente para reducir el sesgo que surge del error de no uniformidad de los mismos. El tamaño del efecto [TE, límite de confianza (LC) 90\%] según la propuesta de (Cohen, 1988), posteriormente adaptada (Hopkins, Marshall, Batterham, \& Hanin, 2009), fue calculado para comparar la magnitud de las diferencias entre el pre-test y el post-test dentro de cada grupo, al igual que para la comparación entre grupos. Los rangos establecidos para los valores del TE fueron $<0.2$ (trivial), > 0.2 (pequeño), > 0.6 (moderado), y > 1.2 (gran- de) (Hopkins et al., 2009). Las diferencias cuantitativas fueron valoradas de manera cualitativa (VC) (Hopkins et al., 2009) estableciendo las siguientes probabilidades: $<1 \%$, casi seguro que no; $1 \%$ a $5 \%$, muy poco probable; $5 \%$ a $25 \%$, poco probable; $25 \%$ a $75 \%$, posible; $75 \%$ a $95 \%$, probable; $95 \%$ a $99 \%$, muy probable; y > 99\%, extremadamente probable. Se determinó un efecto sustancial a diferencias con una probabilidad $>75 \%$ al igual que en estudios previos (Suarez-Arrones et al., 2014). Si se producía la posibilidad de tener resultados beneficiosos/mejores y perjudiciales/peores $>5$, la diferencia real se evaluó como poco claro. Dos hojas de Excel específicas y obtenidas de sportsci.org se utilizaron para analizar diferencias entre-grupos (xCompare2groups. $\mathrm{xls)}$ e intra-grupos (xPostOnlyCrossover.xls).

\section{Resultados}

No se obtuvieron diferencias inter-grupos en ninguna de las variables de rendimiento evaluadas durante el pre-test. El análisis intra-grupo mostró mejoras sustanciales en el GER únicamente en la capacidad de salto ( $\mathrm{CMJ}$ : extremadamente probable moderado) mientras que el GC no mejoró ninguna de las variables objeto de estudio (Tabla 2).

La Figura 1 muestra las diferencias inter-grupos en las diversas variables de rendimiento analizadas. Los resultados indicaron mejoras sustancialmente mayores en CMJ (muy probable grande) en GER respecto GC. En las variables relacionadas con el RSA y con la capacidad de reacción no se observaron diferencias inter-grupos. 


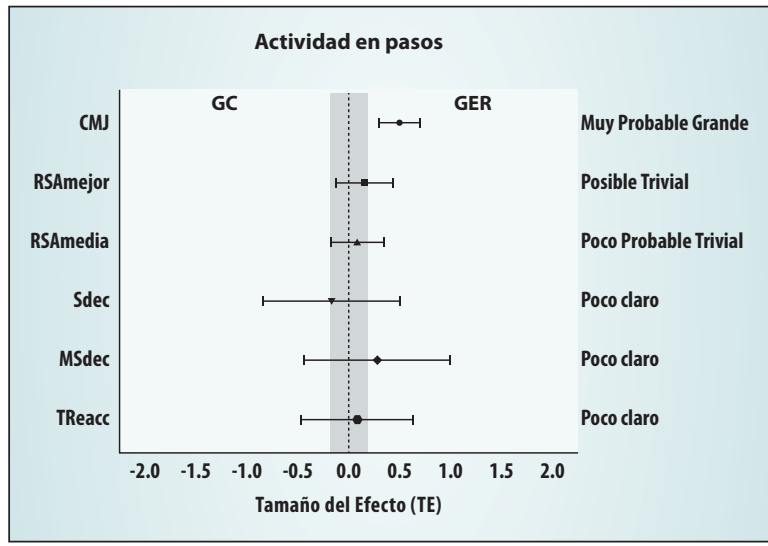

Figura 1. Tamaño del efecto del programa de entrenamiento mediante sprint repetidos (GER) en comparación con el grupo control (GC) en la mejora del salto con contramovimiento (CMJ), tiempo en la mejor repetición ( $\left(\mathrm{RA}_{\text {mejor }}\right)$, tiempo medio $\left(\mathrm{RSA}_{\text {media }}\right.$ ), porcentaje de decrecimiento (Sdec), y porcentaje de decrecimiento teniendo en cuenta el mejor sprint de ambas valoraciones (MSdec) en un test RSA y tiempo de reacción (TReacc) antes de cada uno de los sprints que conforman el test RSA.

Los valores del tiempo de reacción en la valoración pre y post intervención en las repeticiones 1 y 8 fueron de $.760 \pm .094 \mathrm{~ms}^{-1}$ vs. $.731 \pm .065 \mathrm{~ms}^{-1}$ y $.704 \pm$ $.105 \mathrm{~ms}^{-1}$ vs. $.721 \pm .092$ para GER, y $.686 \pm .104 \mathrm{~ms}^{-1}$ vs. $.658 \pm .121 \mathrm{~ms}^{-1}$, y .689 $\pm .159 \mathrm{~ms}^{-1}$ vs. .693 \pm .112 $\mathrm{ms}^{-1}$ para GC, respectivamente. Solo se mostraron diferencias sustanciales entre la valoración pre y post intervención en la tercera repetición ( $\mathrm{TE}=-0.54$; probablemente pequeño) en el grupo GER. Ningún valor de tiempo de reacción mostró diferencias entre ambas valoraciones en GC.

\section{Discusión}

El principal objetivo de este estudio fue analizar los efectos de un entrenamiento basado en sprint repetidos, aplicado durante las cuatro últimas semanas de la temporada competitiva en jugadores jóvenes de fútbol, sobre el RSA, el tiempo de reacción previa a la realización de los esfuerzos de un test RSA y la capacidad de salto. El entrenamiento RST mostró mejoras (TE = .63; 9 \%) en la capacidad de salto. Sin embargo, los resultados del estudio no mostraron efectos positivos de la intervención basada en sprint repetidos en la mejora de las variables determinantes del rendimiento en test RSA como el RSA ${ }_{\text {media }}$ (TE = -.17; posible trivial) y el Sdec (TE = -.11; poco claro), ni del tiempo de reacción ( $\mathrm{TE}=.27$; poco claro).

Contrariamente a nuestra hipótesis, GER y GC no obtuvieron mejoras significativas ni en las variables neuromusculares ni en las metabólicas tras un entrenamiento específico de fútbol con y sin entrenamiento suplementario de RST. Sin embargo, diferentes estu- dios han obtenido mejoras en el rendimiento RSA tras la aplicación de protocolos basados en RST (Buchheit, Méndez-Villanueva, Delhomel, et al., 2010; Ferrari Bravo et al., 2008; Iaia et al., 2015). Estas mejoras pueden ser debidas al llamado efecto aprendizaje, provocado por la relación entre el entrenamiento de sprint y las características del test RSA (Buchheit, 2012). El incremento en la capacidad RSA también puede ser consecuencia de la mejora en la eficiencia energética, la capacidad de recuperación o la tolerancia al lactato (Girard et al., 2011). Es posible que la suplementación con RST en GER ocasionase un incremento en la carga de entrenamiento (Shalfawi, Haugen, Jakobsen, Enoksen, \& Tonnessen, 2013; Tonnessen, Shalfawi, Haugen, \& Enoksen, 2011), con la consiguiente mejora en los procesos de adaptación y mejora del rendimiento (Buchheit, 2012). Sin embargo, estudios previos han mostrado que una sesión de RST puede no incrementar la media de intensidad de entrenamiento sobre el entrenamiento general (Nedrehagen \& Saeterbakken, 2015). Por lo tanto, es posible que el volumen de entrenamiento realizado en este estudio no haya sido suficiente para incrementar el rendimiento, tal y como muestran los valores de distancia recorrida asociados a la sesión de mayor volumen de RST (480 m), los cuales son inferiores $(50 \%)$ a los utilizados en otros trabajos similares que si han obtenido mejoras significativas en esta capacidad (Ferrari Bravo et al., 2008).

Otros aspectos que podrían haber influido en estos resultados son el nivel de condición física de los jugadores (Rodríguez-Fernández et al., 2017), que puede estar en relación con el momento de la temporada en el que se aplica el entrenamiento (Impellizzeri et al., 2008). Es posible que, tras 7 meses de entrenamiento y competición, el potencial de adaptación de los jugadores participantes ya haya alcanzado su máximo En este sentido estudios previos han mostrado que el RSA mejora durante la pretemporada, sigue mejorando en la primera parte del período de competición y se mantiene estable durante el último periodo de la temporada (Impellizzeri et al., 2008). Por otra parte, estudios previos han mostrado los efectos positivos en la pretemporada (Ferrari Bravo et al., 2008) y durante el propio periodo competitivo del RST (Buchheit, Méndez-Villanueva, Delhomel, et al., 2010; Iaia et al., 2017). Además, al contrario que lo observado en nuestros resultados, estudios previos han obtenido mejoras significativas $(p=.02 ; \mathrm{TE}=.89)$ en el RSA media $_{\text {}}$ durante la última fase de la temporada (Nedrehagen \& Saeterbakken, 2015). No obstante, los resultados no pueden ser directamente comparados con los de nuestro estudio tanto por el nivel de los jugadores participantes (jóvenes vs amateur), como por el volumen de 
entrenamiento en cada sesión (2 series de 8 repeticiones vs. 3-4 series de 4-6 repeticiones) Por otro lado, la edad se ha mostrado como un condicionante del rendimiento de RSA, siendo necesario tener en cuenta esta variable al comparar el rendimiento en esta capacidad (Mujika, Spencer, Santisteban, Goiriena, \& Bishop, 2009; Sánchez-Sánchez et al., 2019).

Es difícil obtener una conclusión del rendimiento en RSA empleando solo el Sdec (Impellizzeri et al., 2008), ya que la fatiga puede estar condicionada por el sprint inicial (Méndez-Villanueva et al., 2007), siendo

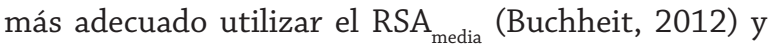
sobre todo contextualizar los valores absolutos e índice de fatiga. Una disminución en el $\mathrm{RSA}_{\text {media }}$ sin cambios en el Sdec se ha asociado a una mejora aneróbica (Ferrari Bravo et al., 2008). En este estudio se llevó a cabo el análisis mediante el MSdec, a partir del cual se determina el decrecimiento teniendo en cuenta el mejor sprint del jugador en el momento óptimo del deportista, independientemente de si este se produce en la valoración pre o post intervención. Este análisis se ha realizado tratando de disminuir la incidencia del sprint inicial en el rendimiento en este test. Ninguno de los dos grupos vio mejorado su rendimiento en el MSdec (TE = -.48; Probable Pequeño y TE = -.59; Probable Pequeño para GER y GC, respectivamente).

Los valores obtenidos en el CMJ son inferiores ( 33 cm vs. $\sim 42 \mathrm{~cm}$ ) a los mostrados por estudios previos para jugadores españoles de similar edad (Sillero et al., 2015). En nuestro estudio hemos obtenido mejoras en el test CMJ tras el entrenamiento RST (TE = .63; Extremadamente Probable Moderado). Estos resultados son contrarios a los de estudios previos (Buchheit, Méndez-Villanueva, Delhomel, et al., 2010), donde no se han obtenido mejoras en el CMJ tras un entrenamiento RST. No obstante, una reciente revisión mostró efectos positivos significativos en ensayos no controlados y controlados del RST sobre el CMJ (Taylor et al., 2015). Los mecanismos que explican estas mejoras se relacionan con el incremento de la fosfocreatina y del glucóneno muscular (Rodas, Ventura, Cadefau, Cusso, \& Parra, 2000), así como los cambios beneficiosos en el patrón de reclutamiento que mejoran el rendimiento neuromuscular (McGill, Chaimberg, Frost, \& Fenwick, 2010).
Uno de los factores que parece afectar al tiempo de reacción es el estrés muscular sufrido (Wilkerson et al., 2017). Además, se ha relacionado dicha capacidad con el riesgo lesional, mostrando disminuciones mediante el entrenamiento de tiempo de reacción con estímulos visuales (Wilkerson et al., 2017). 16 sesiones de entrenamiento mediante saltos con estimulación del tiempo de reacción se han mostrado útiles para la mejora del tiempo de reacción simple (Carvalho Picolini et al., 2015). El protocolo de entrenamiento RST aplicado en nuestro estudio no parece tener efecto (TE = 0.27; poco claro) en la mejora del tiempo de reacción del futbolista durante la realización de un test RSA. Es posible que al no mejorar la resistencia a la fatiga de los jugadores (Sdec) esta se siga manifestando de igual forma en las valoraciones pre y post intervención, condicionando el tiempo de reacción a lo largo de los esfuerzos repetidos. Estudios previos han mostrado que un test RSA similar al utilizado en esta investigación (5 sprints de 30 metros con 20 segundos de recuperación), parece originar fatiga del sistema nervioso central (Clemente Suárez, Muñoz, \& Melús, 2011). Por lo tanto, la ausencia de mejora de la condición física del deportista puede condicionar la respuesta del tiempo de reacción valorado.

\section{Conclusiones}

En conclusión, los resultados obtenidos han mostrado que la suplementación del entrenamiento habitual mediante RST, en las cuatro últimas semanas de la temporada, no determina una mejora útil del RSA, pero sí tiene efectos positivos sobre la capacidad de salto. Es posible que sea necesario un mayor volumen de entrenamiento para mejorar esta capacidad o que en determinados momentos de la temporada, debido al nivel alcanzado por los futbolistas, no sea posible optimizar el rendimiento de los jugadores en la capacidad de repetir sprints. Por lo tanto, podría no ser adecuada su suplementación en periodos próximos al final de la temporada, o cuando el nivel del deportista en esta capacidad es elevado, suponiendo un aumento de la carga de entrenamiento sin ningún efecto en el rendimiento en RSA. 


\section{BIBLIOGRAFÍA}

Aughey, R. J., Hammond, K., Varley, M. C., Schmidt, W. F., Bourdon, P. C., Buchheit, M., ... Gore, C. J. (2013). Soccer activity profile of altitude versus sea-level natives during acclimatisation to $3600 \mathrm{~m}$ (ISA3600). British Journal of Sports Medicine, 47 Suppl 1(Suppl 1), i107-13. doi:10.1136/bjsports-2013-092776

Baker, J., Cote, J., \& Abernethy, B. (2003). Learning from the experts: practice activities of expert decision makers in sport. Research Quarterly for Exercise and Sport, 74(3), 342-347. doi:10.1080/02701367.2 003.10609101

Balsalobre-Fernández, C., Glaister, M., \& Lockey, R. A. (2015). The validity and reliability of an iPhone app for measuring vertical jump performance. Journal of Sports Sciences, 33(15), 1574-1579. doi:10.1080 /02640414.2014.996184

Barnes, C., Archer, D. T., Hogg, B., Bush, M., \& Bradley, P. S. (2014) The evolution of physical and technical performance parameters in the English Premier League. International Journal of Sports Medicine, 35(13), 1095-1100. doi:10.1055/s-0034-1375695

Bishop, D., Girard, O., \& Méndez-Villanueva, A. (2011). Repeated-sprint ability - part II: recommendations for training. Sports Med, 41(9), 741-756. doi:10.2165/11590560-000000000-00000

Buchheit, M, \& Laursen, P. B. (2013). High-intensity interval training, solutions to the programming puzzle: Part I: cardiopulmonary emphasis. Sports Med, 43(5), 313-338. doi:10.1007/s40279-013-0029-x

Buchheit, M. (2012). Should we be recommending repeated sprints to improve repeated-sprint performance? Sports Med, 42(2), 163-169. doi:10.2165/11598230-000000000-00000

Buchheit, M, Méndez-Villanueva, A., Delhomel, G., Brughelli, M., \& Ahmaidi, S. (2010). Improving repeated sprint ability in young elite soccer players: repeated shuttle sprints vs. explosive strength training. J Strength Cond Res, 24(10), 2715-2722. doi:10.1519/JSC.0b013e$3181 b f 0223$

Buchheit, M, Méndez-villanueva, A., Simpson, B. M., \& Bourdon, P. C. (2010). Repeated-sprint sequences during youth soccer matches. Int $J$ Sports Med, 31(10), 709-716. doi:10.1055/s-0030-1261897

Buchheit, M, Méndez-Villanueva, A., Simpson, B. M., \& Bourdon, P. C. (2010). Match running performance and fitness in youth soccer. International Journal of Sports Medicine, 31(11), 818-825. doi:10.1055/ s-0030-1262838

Campos-Vazquez, M. A., Romero-Boza, S., Toscano-Bendala, F. J., Leon-Prados, J. A., Suarez-Arrones, L. J., \& Gonzalez-Jurado, J. A. (2015). Comparison of the effect of repeated-sprint training combined with two different methods of strength training on young soccer players. J Strength Cond Res, 29(3), 744-751. doi:10.1519/ JSC.0000000000000700

Carvalho Picolini, L., Saraiva Flôres, F., dos Santos Soares, E., Lopes dos Santos, D., Corrêa Matheus, S., \& Corazza, S. (2015). Efeitos da prática de Jump no equilíbrio e no tempo de reação em mulheres. ConScientiae Saúde, 14(4), 585-591.

Chaouachi, A., Manzi, V., Wong del, P., Chaalali, A., Laurencelle, L., Chamari, K., \& Castagna, C. (2010). Intermittent endurance and repeated sprint ability in soccer players. J Strength Cond Res, 24(10), 2663-2669. doi:10.1519/JSC.0b013e3181e347f4

Clemente Suárez, V., Muñoz, V. E., \& Melús, M. (2011). Fatiga del sistema nervioso después de realizar un test de capacidad de sprint repetidos (RSA) en jugadores de fútbol profesionalres. Archivos de Medicina Del Deporte, 28(143), 174-180.

Cohen, J. (1988). Statistical Power Analysis for the Behavioral Sciences. Hillsdale, N.J: L. Erlbaum Associates.

Eckner, J. T., Kutcher, J. S., \& Richardson, J. K. (2010). Pilot evaluation of a novel clinical test of reaction time in national collegiate athletic association division I football players. Journal of Athletic Training, 45(4), 327-332. doi:10.4085/1062-6050-45.4.327

Eniseler, N., Şahan, Ç., Özcan, I., \& Dinler, K. (2017). High-Intensity Small-Sided Games versus Repeated Sprint Training in Junior Soc cer Players. Journal of Human Kinetics, 60(1), 101-111. doi:10.1515/ hukin-2017-0104

Faude, O., Koch, T., \& Meyer, T. (2012). Straight sprinting is the most frequent action in goal situations in professional football. J Sports Sci, 30(7), 625-631. doi:10.1080/02640414.2012.665940

Ferrari Bravo, D., Impellizzeri, F. M., Rampinini, E., Castagna, C., Bishop, D., \& Wisloff, U. (2008). Sprint vs. interval training in football. Int $J$ Sports Med, 29(8), 668-674. doi:10.1055/s-2007-989371

Fransson, D., Nielsen, T. S., Olsson, K., Christensson, T., Bradley, P. S., Fatouros, I. G., ... Mohr, M. (2018). Skeletal muscle and performance adaptations to high-intensity training in elite male soccer players: speed endurance runs versus small-sided game training. European Journal of Applied Physiology, 118(1), 111-121. doi:10.1007/s00421-017-3751-5

Gabbett, T. J., Kennelly, S., Sheehan, J., Hawkins, R., Milsom, J., King, E., ... Ekstrand, J. (2016). If overuse injury is a "training load error", should undertraining be viewed the same way? British Journal of Sports Medicine, 50(17), 1017-1018. doi:10.1136/bjsports-2016-096308

Girard, O., Méndez-Villanueva, A., \& Bishop, D. (2011). Repeated-sprint ability part I: Factors contributing to fatigue. Sports Medicine. doi:10.2165/11590550-000000000-00000

Hopkins, W. G., Marshall, S. W., Batterham, A. M., \& Hanin, J. (2009) Progressive statistics for studies in sports medicine and exercise science. Medicine and Science in Sports and Exercise, 41(1), 3-13. doi:10.1249/MSS.0b013e31818cb278

Iaia, F., Fiorenza, M., Larghi, L., Alberti, G., Millet, G., \& Girard, O. (2017) Short-or long-rest intervals during repeatedsprint training in soccer? PLoS ONE, 12(2), e0171462. doi:10.1371/journal.pone.0171462

Iaia, F., Fiorenza, M., Perri, E., Alberti, G., Millet, G. P., \& Bangsbo, J. (2015). The Effect of Two Speed Endurance Training Regimes on Performance of Soccer Players. PLoS One, 10(9), e0138096. doi:10.1371/ journal.pone.0138096

Impellizzeri, F. M., Rampinini, E., Castagna, C., Bishop, D., Ferrari Bravo, D., Tibaudi, A., \& Wisloff, U. (2008). Validity of a repeated-sprint test for football. Int J Sports Med, 29(11), 899-905. doi:10.1055/s-2008-1038491

McGill, S. M., Chaimberg, J. D., Frost, D. M., \& Fenwick, C. M. J. (2010). Evidence of a double peak in muscle activation to enhance strike speed and force: an example with elite mixed martial arts fighters. Journal of Strength and Conditioning Research, 24(2), 348-357. doi:10.1519/ JSC.0b013e3181cc23d5

Méndez-Villanueva, A., Hamer, P., \& Bishop, D. (2007). Physical fitness and performance. Fatigue responses during repeated sprints matched for initial mechanical output. Med Sci Sports Exerc, 39(12), 22192225. doi:10.1249/mss.0b013e31815669dc

Méndez-Villanueva, A., Hamer, P., \& Bishop, D. (2008). Fatigue in repeated-sprint exercise is related to muscle power factors and reduced neuromuscular activity. European Journal of Applied Physiology, 103(4), 411-9. doi:10.1007/s00421-008-0723-9

Miller, J. O., \& Low, K. (2001). Motor processes in simple, go/no-go, and choice reaction time tasks: a psychophysiological analysis. Journal of Experimental Psychology. Human Perception and Performance, 27(2), 266-289.

Mujika, I., Spencer, M., Santisteban, J., Goiriena, J. J., \& Bishop, D. (2009). Age-related differences in repeated-sprint ability in highly trained youth football players. J Sports Sci, 27(14), 1581-1590. doi:10.1080/02640410903350281

Nedrehagen, E. S., \& Saeterbakken, A. H. (2015). The Effects of in-Season Repeated Sprint Training Compared to Regular Soccer Training. $J$ Hum Kinet, 49, 237-244. doi:10.1515/hukin-2015-0126

Owen, A., Lago-Peñas, C., Gómez, M.-Á., Mendes, B., \& Dellal, A. (2017). Analysis of a training mesocycle and positional quantification in elite European soccer players. International Journal of Sports Science \& Coaching, 12(5), 174795411772785. doi:10.1177/1747954117727851

Owen, A., Wong del, P., Paul, D., \& Dellal, A. (2012). Effects of a periodized small-sided game training intervention on physical performance in elite professional soccer. J Strength Cond Res, 26(10), 27482754. doi:10.1519/JSC.0b013e318242d2d1

Pereira Da Silva, N., Kirkendall, D. T., \& Leite De Barros Neto, T. (2007) Movement patterns in elite Brazilian youth soccer. The Journal of Sports Medicine and Physical Fitness, 47(3), 270-275. 
Rampinini, E., Bishop, D., Marcora, S., Ferrari Bravo, D., Sassi, R., \& Impellizzeri, F. (2007). Validity of simple field tests as indicators of match-related physical performance in top-level professional soccer players. Int J Sports Med, 28(3), 228-235. doi:10.1055/s-2006-924340 Rampinini, E., Coutts, A. J., Castagna, C., Sassi, R., \& Impellizzeri, F. M. (2007). Variation in top level soccer match performance. Int J Sports Med, 28(12), 1018-1024. doi:10.1055/s-2007-965158

Rampinini, E., Sassi, A., Morelli, A., Mazzoni, S., Fanchini, M., \& Coutts, A. J. (2009). Repeated-sprint ability in professional and amateur soccer players. Appl Physiol Nutr Metab, 34(6), 1048-1054. doi:10.1139/ H09-111

Rodas, G., Ventura, J. L., Cadefau, J. A., Cusso, R., \& Parra, J. (2000). A short training programme for the rapid improvement of both aerobic and anaerobic metabolism. Eur J Appl Physiol, 82(5-6), 480-486. doi:10.1007/s004210000223

Rodríguez-Fernández, A., Sánchez Sánchez, J., Rodríguez-Marroyo, J. A., Casamichana, D., \& Villa, J. G. (2017). Effects of 5-week pre-season small-sided-game-based training on repeat sprint ability. The Journal of Sports Medicine and Physical Fitness, 57(5), 529-536. doi:10.23736/ S0022-4707.16.06263-0

Rodríguez Fernández, A., Sánchez Sánchez, J., \& Villa Vicente, J. G. (2014). Efectos de 2 tipos de entrenamiento interválico de alta intensidad en la habilidad para realizar esfuerzos máximos (RSA) durante una pretemporada de fútbol. Cultura, Ciencia y Deporte, 9(27), 251259. doi:10.12800/ccd.v9i27.467

Sánchez-Sánchez, J., García-Unanue, J., Hernando, E., López-Fernández, J., Colino, E., León-Jiménez, M., \& Gallardo, L. (2019). Repeated Sprint Ability and Muscular Responses According to the Age Category in Elite Youth Soccer Players. Frontiers in Physiology, 10(June). doi:10.3389/fphys.2019.00175

Schwab, S., \& Memmert, D. (2012). The impact of a sports vision training program in youth field hockey players. Journal of Sports Science \& Medicine, 11(4), 624-631.
Shalfawi, S. A., Haugen, T., Jakobsen, T. A., Enoksen, E., \& Tonnessen, E. (2013). The effect of combined resisted agility and repeated sprint training vs. strength training on female elite soccer players. J Strength Cond Res, 27(11), 2966-2972. doi:10.1519/JSC.0b013e31828c2889

Sillero, B., Silva-Grigoletto, D., Herrera, M., Montero, M., Guillén, A. Y., Castillo, D., \& Guillén, Y. (2015). Capacidades físicas en jugadores de fútbol formativo de un club profesional. Revista Internacional de $\mathrm{Me}$ dicina y Ciencias de La Actividad Física y El Deporte, 15(58), 289-307. doi:10.15366/rimcafd2015.58.006

Suarez-Arrones, L., Tous-Fajardo, J., Nunez, J., Gonzalo-Skok, O., Galvez, J., \& Méndez-Villanueva, A. (2014). Concurrent repeated-sprint and resistance training with superimposed vibrations in rugby players. International Journal of Sports Physiology and Performance, 9(4), 667-673. doi:10.1123/ijspp.2013-0238

Taylor, J., Macpherson, T., Spears, I., \& Weston, M. (2015). The effects of repeated-sprint training on field-based fitness measures: a meta-analysis of controlled and non-controlled trials. Sports Med, 45(6), 881-891. doi:10.1007/s40279-015-0324-9

Tonnessen, E., Shalfawi, S. A., Haugen, T., \& Enoksen, E. (2011). The effect of 40-m repeated sprint training on maximum sprinting speed, repeated sprint speed endurance, vertical jump, and aerobic capacity in young elite male soccer players. J Strength Cond Res, 25(9), 23642370. doi:10.1519/JSC.0b013e3182023a65

Vigh-Larsen, J. F., Dalgas, U., \& Andersen, T. B. (2018). Position-Specific Acceleration and Deceleration Profiles in Elite Youth and Senior Soccer Players. Journal of Strength and Conditioning Research, 32(4), 1114-1122. doi:10.1519/JSC.0000000000001918

Wilkerson, G. B., Simpson, K. A., \& Clark, R. A. (2017). Assessment and Training of Visuomotor Reaction Time for Football Injury Prevention. Journal of Sport Rehabilitation, 26(1), 26-34. doi:10.1123/jsr.20150068 